\title{
¿Tesis o examen de grado? Un dilema para la formación investigativa
}

\section{Thesis or degree-by-examination? A Dilemma for Research Training}

\begin{abstract}
Armando Romero-Ortega es Docente titular de la Universidad Politécnica Salesiana de Quito (Ecuador) (aromero@ups.edu.ec) (http://orcid.org/0000-0001-6904-9353)

Dra. Teresa Sanz-Cabrera es Docente titular de la Universidad de la Habana (Cuba) (teresa@cepes.uh.cu) (http://orcid.org/0000-000 1-9794-5315)
\end{abstract}

Recibido: 2017-01-05 / Revisado: 2017-03-31 / Aceptado: 2017-04-24 / Publicado: 2017-07-01

\section{Resumen}

El nivel inicial del sistema educativo ecuatoriano urge de docentes con una sólida formación investigativa que les permita incidir de manera positiva en los nuevos contextos educativos. Un factor vinculado a este proceso es la elaboración del trabajo de titulación o el examen de grado que el estudiante debe rendir previo a su graduación. El objetivo de este estudio es determinar en qué medida se afecta la formación investigativa de los estudiantes de la Mención Parvularia de la Universidad Politécnica Salesiana con la aplicación de la nueva normativa que incluye el examen de grado como una alternativa al trabajo de titulación. Para ello se analiza el proceso de graduación de esta carrera en el período académico (2015-2016) a partir de algunos principios de la pedagogía crítica. Los datos revelan, por un lado, el incremento de la eficiencia terminal con la aplicación de los exámenes de grado; y, por otro, la disminución de experiencias de carácter investigativo propias de los trabajos de titulación, con lo cual se ve afectada la for- mación investigativa de los futuros docentes parvularios. La conclusión a la que se llega es que esta formación debe ser re-significada por la carrera y dentro de ella, el trabajo de titulación necesita ser repotenciado para que cumpla sus objetivos educativos.

Descriptores: Formación investigativa, trabajo de titulación, examen de grado, graduación, pedagogía crítica, acreditación.

\footnotetext{
Abstract

Nursery Schools in the ecuadorian educational system urges teachers with a solid research training that allows them to have a positive impact on the new educational contexts. One factor linked to this process is the preparation of the degree work or the degree examination that the student must perform prior to graduation. The aim of this study is to determine the extent to which the research training of Nursery School at the Universidad Politécnica Salesiana is affected by the application of the new regulations that include a final
} 
test as an alternative to a research in order to obtain an undergraduate degree. For this, the process of graduation of this career in the academic period (2015-2016) is analyzed through some principles of critical pedagogy. The data reveal, on one hand, the increase of efficiency with the application of the exams of degree; and, on the other, the reduction of investigative experiences of the degree work, which is affecting the research training of future nursery teachers. The conclusion reached is that research training must be signified by the career and within it the research work needs to be repotenced to fulfill its educational goals.

Keywords: Research training, research work, degree examination, graduation, critical pedagogy, accreditation.

\section{Introducción}

Por décadas la universidad ecuatoriana ha exigido como requisito para la graduación de los futuros profesionales, la realización de un trabajo académico en el que se evidencie el dominio teórico y metodológico del área disciplinar del graduado. Varios son los nombres que se han otorgado a este trabajo: tesis, tesina, monografía, trabajo de grado, trabajo de titulación, entre otros. En el caso de la Mención Parvularia de la Universidad Politécnica Salesiana de Ecuador (UPS), donde se realiza este estudio, desde sus inicios en el año 1994 hasta el 2015, quienes aspiraban obtener el título de docentes parvularios podían optar por la realización de una tesis, un proyecto o un producto, siendo la primera la que mayores dificultades de índole teórico presentaba. Los proyectos y los productos, en cambio, se orientaban más hacia propuestas de tipo metodológico. En dicho contexto, las preferencias de los estudiantes se inclinaban hacia estas últimas alternativas, quedando las tesis restringidas a un número cada vez menor de interesados. Eso daba cuenta de alguna disfunción en el proceso relacionado con la formación investigativa de los futuros docentes parvularios. Esta situación vivida por la Mención Parvularia, no era la excepción, sino una muestra de una tendencia general.

En efecto, la baja eficiencia terminal de los estudiantes, es una de las grandes deudas de la universidad ecuatoriana, según Larrea (2016), en el año 2013 sólo once de cada 100 universitarios lograba graduarse en los plazos establecidos. Esta tendencia también se replica en la UPS, cuyo índice promedio de graduación se ubicaba en el mismo año en el 8,39\% (UPS, 2013). Tratándose de un tema delicado y recurrente, años atrás la UPS implementó algunas iniciativas con el fin de mejorar este indicador, entre éstas se encuentran los cursos de graduación, que eran diseñados e implementados con el fin de graduar a la población estudiantil que por diversas razones no lograba terminar este proceso. También está la creación del centro de elaboración de productos de grado, cuyo propósito era guiar y orientar metodológicamente ya sea las tesis, los proyectos o los productos que los estudiantes debían elaborar para graduarse. Estas experiencias, a más de aumentar la tasa de graduación, permitieron ubicar el problema de la baja eficiencia terminal vinculado no sólo a los procesos administrativos, sino también a la formación investigativa que recibían los estudiantes. Es decir que los esfuerzos no debían concentrarse solamente en la etapa final de la carrera, la graduación, sino -sobre todo- en el proceso de enseñanza-aprendizaje de los conocimientos, habilidades y valores vinculados al ejercicio investigativo que se debe desarrollar en todos los niveles de la formación profesional.

Este escenario vinculado al proceso de graduación de los estudiantes universitarios que había permanecido inalterado por largo tiempo se vio significativamente afectado en el año 2015 con la entrada en vigencia de un nuevo reglamento académico emitido por el Consejo de Educación Superior del Ecuador (CES). En este instrumento legal se amplían las opciones para la graduación de los estudiantes. De las tres anteriormente nombradas en el caso de Parvularia de la UPS, se 
extienden a 15 las posibilidades para los trabajos de titulación, y si ninguna de ellas satisface las expectativas del estudiante, éste puede optar por el examen de grado de carácter complexivo (CES, 2015b). El trabajo de titulación se considera en este reglamento, en su art. 21, numeral 3, como una "propuesta innovadora que contiene, como mínimo, una investigación exploratoria y diagnóstica, base conceptual, conclusiones y fuentes de consulta" (CES, 2015a), mientras que para el examen de grado se menciona que deberá ser de "carácter complexivo, articulado al perfil de egreso de la carrera, con el mismo nivel de complejidad, tiempo de preparación y demostración de resultados de aprendizaje o competencias, que el exigido en las diversas formas del trabajo de titulación" (Ídem). La Mención Parvularia de la UPS escoge, de entre las 15 opciones planteadas por el CES para el trabajo de titulación, el análisis de caso y las propuestas metodológicas y, como se podrá colegir, también el examen de grado (UPS, 2016).

Habiendo advertido la poca aceptación que tenía la elaboración de una tesis entre los estudiantes de Parvularia, no es de extrañar que en el nuevo escenario planteado por la normativa académica de reciente aplicación, la gran mayoría de estudiantes opten por el examen de grado. Pareciera que este comportamiento está vinculado a la percepción que los futuros docentes parvularios tienen sobre el examen de grado en relación al trabajo de titulación, al considerar al primero como más fácil, más rápido y menos estresante que el segundo. Este cambio, que a simple vista pareciera no tener mayores repercusiones, reviste algunas implicaciones significativas para la formación investigativa. Los estudiantes, al optar por el examen de grado, se privan de una experiencia investigativa única durante su proceso formativo como es la realización de un trabajo de titulación, pues, desde la perspectiva de la formación investigativa, esta modalidad es la que mayores aportes ofrece. La adquisición de las capacidades investigativas, claro está, no depende únicamente de los trabajos de titulación, sino que es un proceso que se da a lo largo de toda la formación universitaria y se encuentra presente en casi todas las actividades académicas dentro o fuera del aula.

Lo ocurrido en el último período da cuenta de una situación paradójica en la formación investigativa de la Mención Parvularia; por un lado, la tasa de graduación casi se duplica en los últimos períodos y, por otro, los proyectos de investigación vinculados al proceso de graduación se reducen significativamente. Esta preocupación cobra mayor relevancia cuando se constata los modestos resultados alcanzados por los estudiantes de esta carrera en cuanto a los aprendizajes vinculados al eje investigativo, como se aprecia en los informes realizados por la comisión de evaluación de la UPS (COMEVAL, 2014; 2015). Si bien es cierto que la elaboración de un trabajo de titulación implica una serie de dificultades para los estudiantes, como no saber qué tema investigar, qué diseño de investigación asumir, cómo elaborar el marco teórico, la falta de tiempo debido a que muchos estudiantes empezaban a trabajar, etc., y que esas circunstancias determinan en buena medida el bajo índice de eficiencia terminal en las universidades de la región, como lo demuestran los trabajos de varios autores (Guevara y Viera, 2009; Gascón, 2008; Ferrer y Malaver, 2000; Abreu, 2015; Larrea, 2014), también es cierto que, cuando esos procesos son bien encaminados, se convierten en potentes espacios para el ejercicio investigativo de los futuros profesionales de la educación. En la elaboración de una tesis, un proyecto o un producto, se imbrican conocimientos, habilidades y valores vinculados al quehacer investigativo (García, 2010; Paulo y Chirino, 2012; Chirino, 2002; Medina \& Salvador, 2009; Guzmán, 2003). En este contexto, el propósito de este trabajo es analizar los impactos que está generando en la formación investigativa de los estudiantes de la Mención Parvularia de la UPS la implementación de la nueva normativa referente a los procesos de graduación.

\section{Metodología}

Este estudio se desprende de un trabajo más amplio que el autor viene desarrollando desde el 
año 2013 con el propósito de entender las concepciones y prácticas relativas a la formación investigativa de los futuros docentes que se gradúan en la UPS. A partir de lo ocurrido en el período académico 2015-2016 con los estudiantes de dos cohortes de la Mención Parvularia, se presentan algunos datos empíricos para dimensionar el problema y se proponen breves pistas teóricas para su comprensión desde la pedagogía crítica.

\section{El proceso de transición del sistema de educación superior del Ecuador}

La problemática descrita en los párrafos precedentes debe ser entendida en el contexto de transición que está viviendo la universidad ecuatoriana (Ponce, 2016; Fabara, 2013), el cual obedece a una tendencia global y homogeneizante de la educación superior (UNESCO, 1998; Buendía, 2007; Tuning, 2007). Para el caso ecuatoriano, este proceso ha implicado un nuevo marco legal y la implementación de evaluaciones, acreditaciones y categorizaciones de las instituciones de educación superior que trae como resultado, en la primera fase, el cierre de 14 universidades por no cumplir con los requisitos exigidos para su funcionamiento; se categorizan a las universidades y las que se encuentran en los últimos lugares deben elaborar sus respectivos planes de mejoramiento; se regula el ingreso de estudiantes a la universidad a través de la meritocracia; se elevan los estándares para las evaluaciones y acreditaciones institucionales; se crean tres universidades públicas que pretenden convertirse en modelo para las demás; se exige el rediseño de los proyectos curriculares de las carreras de grado, empezando por educación (Ponce, 2016). Estos y otros elementos vinculados a la búsqueda de la calidad en la educación superior imprimieron en la universidad ecuatoriana una nueva dinámica marcada por una mezcla de optimismo e incertidumbre, pues a pesar de que estos cambios eran esperados y necesarios, las preocupaciones pronto surgieron por los breves plazos previstos y los mecanismos establecidos para su implementación.

De un sistema educativo anterior, que pretendía estimular la autorregulación, se pasa a otro donde la intervención del Estado cobra un nuevo protagonismo. Este fenómeno se encuentra estrechamente ligado al proceso político que el país ha vivido en los últimos 10 años en donde la universidad es considerada como un factor clave para el desarrollo (SENPLADES, 2013). En el esquema anterior, al ser la autorregulación mal entendida y aplicada, tanto por el sector público como el privado, se cometieron una serie de excesos por parte de algunas instituciones dando lugar a lo que en su momento se denominaron las "universidades de garaje" (CONEA, 2009). En la actualidad el panorama ha cambiado significativamente, es el Estado el que asume las riendas de la educación superior y con ello empieza un nuevo ciclo que no está exento de ciertos riesgos. No es exagerado afirmar que, durante varios momentos de la vida institucional la principal preocupación de los directivos ha sido la de demostrar a los organismos de control que se están cumpliendo con los indicadores de gestión, requisito indispensable para la posterior acreditación. Es en este contexto que la Universidad Politécnica Salesiana ha debido adoptar un nuevo estilo de trabajo caracterizado por los planes de mejora, los planes operativos anuales, los informes de evaluación y sus respectivas evidencias que son requeridos periódicamente por los organismos de control como el Consejo de Educación Superior (CES) y el Consejo de Evaluación, Acreditación y Aseguramiento de la Calidad de la Educación Superior (CEAACES), donde los indicadores y evidencias adquieren un protagonismo notable. Uno de estos indicadores es la tasa de eficiencia terminal.

\section{El caso de la Mención Parvularia de la UPS}

Dentro de la nueva cultura de calidad educativa que se está implementando en el país, el mejoramiento de los indicadores se ha convertido en 
una prioridad institucional, entre los cuales se encuentra el indicador tasa de graduación, entendida como el "número de estudiantes graduados en un período académico" (UPS, 2015, p. 94). En el actual modelo de evaluación institucional del Consejo de Evaluación, Acreditación y Aseguramiento de la Calidad de la Educación Superior, la tasa de titulación o graduación es un indicador del subcriterio eficiencia académi$\mathrm{ca}$, con un peso de 0,0250 puntos en la escala de valoración conformada por 44 indicadores (CEAACES, 2015), lo que determina que se le preste especial atención a este indicador. Por su parte, el Consejo de Educación Superior, con el fin de aumentar el número de graduados a nivel nacional, incluyó en el nuevo reglamento de régimen académico, en su disposición transitoria quinta, literal a), las Unidades de Titulación Especial (CES, 2015a). Estas Unidades asumieron con gran dinamismo su cometido, logrando reducir de manera significativa el índice de estudiantes egresados que no conseguían graduarse.

En el período 46 (marzo-agosto de 2015), la Unidad de Titulación Especial de la Mención Parvularia debió implementar las nuevas disposiciones del reglamente académico, pero se encontró con que el número de docentes era insuficiente para cubrir la demanda de estudiantes en proceso de graduación. Para contrarrestar esta deficiencia se tomó la decisión de priorizar el análisis de caso, por cuanto permitía trabajar al mismo docente con varios estudiantes a la vez. Para ello se desarrolló desde la carrera un proyecto investigativo orientado a conocer las prácticas de enseñanza en educación general básica en los centros educativos de la zona. A partir de este proyecto, los estudiantes se ubicaron en instituciones educativas en las cuales realizaron los análisis de caso con la guía de su respetivo tutor. Como resultado de esta iniciativa nueva en la carrera, el 80\% de estudiantes optó por el análisis de caso y el restante $20 \%$ se inclinó por el examen de grado. La experiencia resultó positiva y alentadora; sin embargo, la carencia de profesores en el número requerido y los trámites administrativos que aún no estaban totalmente ajustados a los nuevos requerimientos obligó a que en el período siguiente (septiembre 2015-febrero 2016) la Unidad de Titulación Especial diera prioridad a los exámenes de grado, de tal forma que los datos respecto a las opciones de graduación casi se invierten en relación al período anterior; en esta segunda experiencia, el $88 \%$ de estudiantes optaron por el examen y solo el $12 \%$ lo hizo por el trabajo de titulación. Como resultado de estas acciones se tiene que del 12,50 \% del año 2014, la tasa de graduación se incrementó al 23,48 \% para el año siguiente (UPS, 2015).

Estas cifras cuantitativas resultan bastante halagadoras desde la perspectiva de la evaluación y acreditación institucional; pero no ocurre lo mismo si se miran con detenimiento y a profundidad desde una perspectiva pedagógica crítica. Ante ello surge la pregunta ¿cómo mejorar la tasa de graduación sin afectar la formación investigativa de los estudiantes de la Mención Parvularia de la UPS? Se ha pasado de un extremo a otro. En el modelo anterior, había más trabajos investigativos elaborados por los estudiantes, pero pocos graduados; en el modelo actual, hay más graduados, pero hay menos investigaciones en la carrera. En ninguno de los dos extremos se puede hablar de una adecuada formación investigativa. ¿Es posible el equilibrio? A continuación se presentan algunos elementos teóricos para entender la dimensión de esta situación.

\section{La formación investigativa desde la pedagogía crítica}

La tarea docente siempre ha estado atravesada por una serie de elementos que la vuelven compleja, y más aún en la época actual, caracterizada por los vertiginosos cambios, las tendencias globalizadoras, los retos generados por las diferencias culturales, sociales y de género, etc. Se requiere un docente capaz de problematizar, de indagar, de formular hipótesis, de buscar soluciones a sus problemas educativos cotidianos; en suma, se requiere un docente investigador transformador 
(Freire, 2001; Schön, 1992; Carr y Kemmis, 1988). En el ámbito de la investigación educativa aún persiste en algunos sectores la imagen del investigador como el intelectual que se encuentra en la frontera del conocimiento, con una formación super-especializada que lo convierte en experto de los temas educativos, pero poco vinculado al mundo cotidiano. En el otro extremo se encuentra la figura del docente, del práctico (Giroux, 1990), quien debe lidiar a diario en el aula con una serie de problemas para los que no siempre tiene la solución esperada. En esta visión se presenta un enorme abismo entre el investigador y el docente. De lo que se trata es de apostar por un nuevo docente que conjugue en su práctica la unidad dialéctica de la teoría y la práctica (Carr y Kemmis, 1988; Carr, 2002); un docente con una sólida formación pedagógica y con capacidades para investigar su práctica docente en relación con el contexto en que se encuentre; un docente que, a través del método científico, se acerque a la realidad y busque su transformación porque está convencido que su acción es parte de una cadena de intervenciones que buscan una sociedad más armónica y equilibrada (McLaren, 1994; Ordóñez, 2002; Ramírez, 2008). Es allí donde cobra pleno sentido la formación investigativa de los futuros docentes. Esta formación debe estar sustentada en los principios educativos institucionales que para el caso de la UPS se declara en su misión: "el desafío de nuestra propuesta educativa liberadora es formar actores sociales y políticos con una visión crítica de la realidad, socialmente responsables, con voluntad transformadora y dirigida de manera preferencial a los pobres" (UPS, 2009, p. 22). Vista de esta manera la formación investigativa, queda claro que su ámbito de injerencia y de interrelaciones rebasa a las asignaturas del área de investigación y se convierte en un eje transversal que debe ser abordado por todas las asignaturas y los espacios curriculares de la carrera (UPS, 2016). La investigación se convierte entonces en el eje articulador de la teoría con la práctica.

\section{Tendencias en la formación investigativa}

En relación al proceso formativo de las capacidades investigativas de los estudiantes vinculados a las carreras de educación, algunas tendencias se han notado en el contexto latinoamericano. Una de ellas es ubicar la formación investigativa en los niveles de posgrado; otra opta por incluir dentro del currículo de grado disciplinas o asignaturas cuya finalidad es brindar herramientas de metodología de la investigación (Rojas \& Aguirre, 2015), éste es el caso de la Mención Parvularia de la UPS. Una tercera vía es el trabajo extracurricular que se desarrolla en paralelo, por ejemplo las experiencias de los semilleros de investigación en Colombia (Quintero-Corzo, Molina, \& MunévarQuintero, 2008). Obviamente existen otras alternativas, pero éstas son las más expandidas, pero todas procuran fortalecer la formación investigativa y para ello hacen falta intervenciones en los tres niveles del currículo. La formación investigativa no puede reducirse a capacitar a los estudiantes para que elaboren exitosamente su trabajo de grado por medio de asignaturas de metodología de la investigación. Se trata, más bien, de un proceso continuo donde se desarrollan conocimientos, habilidades y valores de la actividad científica que posibilitan en el educador un nuevo nivel de desarrollo integral para actuar en la práctica y transformarla (García, 2010). El trabajo de grado, en este contexto, viene a ser el espacio privilegiado para integrar estos elementos, ya que los estudiantes y docentes disponen del tiempo, herramientas e insumos para ello, situación que difícilmente se da en otras actividades académicas como es el caso de los exámenes de grado. De allí la importancia de que la carrera recupere este valioso espacio para la investigación. Ya no se trata de cumplir únicamente con un requisito para graduarse, como lo ven muchos estudiantes, sino la posibilidad real de llevar a la esfera de la práctica el acervo de conocimientos teóricos recibidos en las diferentes asignaturas establecidas en el currículo. Estas experiencias adquieren un 
salto cualitativo cuando los estudiantes se vinculan a proyectos de investigación que desarrollan docentes de la carrera, como en efecto ya se hado en algunos casos y con resultados sumamente alentadores porque ciertamente la mejor manera de aprender a investigar es investigando.

\section{Los contenidos de la formación investigativa}

Un aspecto de suma importancia para la formación investigativa es el de los contenidos, tarea nada fácil si no se toman en cuenta criterios de gradualidad, secuenciación, pertinencia e interdisciplinariedad. Medina y Salvador definen las siguientes características para los contenidos: 1). Representan una selección hecha con criterios de racionalidad, eficiencia y funcionalidad de la cultura de una sociedad; 2). Son saberes organizados en disciplinas o asignaturas y adecuados a las características de los alumnos; 3 ). Su asimilación es esencial para la formación integral de los alumnos; 4). No son un fin en sí mismos, sino un paso más en la consecución de las capacidades y competencias básicas; 5). Esta asimilación implica reconstruir y reelaborar el saber y para ello se requiere ayuda específica (2009, p. 143). Estos mismos autores se refieren a los contenidos como "el conjunto de procedimientos, destrezas y habilidades que permiten a los alumnos construir el conocimiento, y, también, el sistema de actitudes, valores y normas que regulan la vida en sociedad" (2009, p. 144). Los contenidos no sólo se refieren a los conocimientos o saberes, sino también a lo procedimental y a lo actitudinal (Díaz-Barriga \& Hernández, 2010), es decir a las habilidades y valores, con los cuales se pretende alcanzar una formación que integre los saberes con el saber hacer y el saber estar. Lograr el equilibrio entre estos tres tipos de contenidos es un reto continuo para la Mención Parvularia. Por los resultados obtenidos y que han sido analizados por el autor en otros trabajos referidos a esta temática, es evidente que se ha dado mayor énfasis a los contenidos cognitivos, quedando los procedimentales y actitudinales relegados a un segundo plano. Y es este desbalance precisamente el que determina el temor que sienten los estudiantes cuando tienen que emprender un proyecto investigativo, al no sentirse seguros de sus capacidades (Esper, 2008; Carreras et al., 2009). Los contenidos cognitivos se olvidan rápida y fácilmente si no tienen la oportunidad de ser incorporados al modo de actuación cotidiano de los estudiantes. Se vuelve imprescindible, entonces, trabajar en enfoques y metodologías que impulsen también el desarrollo de habilidades y valores vinculados a la investigación.

\section{Requerimientos del currículo de educación inicial del Ecuador para la formación investigativa}

En el ámbito del ejercicio profesional del docente parvulario, se debe tomar en cuenta las exigencias del nuevo currículo planteado para la educación inicial del Ecuador en el año 2014. En él se plantean como directrices algunos principios del constructivismo, el buen trato y la interculturalidad. Como características de este currículo están la formación integral del niño, la flexibilidad, la concepción del niño como un ser único e irrepetible y el involucramiento de la familia. Todo lo anterior bien puede resumirse en la visión de que "todos los niños son seres bio-psicosociales y culturales, únicos e irrepetibles y los ubica como actores centrales del proceso de enseñanza aprendizaje" (Ministerio de Educación del Ecuador, 2014, p. 16). Todos estos elementos obligan a la Mención Parvularia a superar la antigua concepción "instrumentalista" del educador parvulario y en su lugar apostar por un docente con sólidos conocimientos pedagógicos y desarrolladas capacidades investigativas que entiendan la problemática educativa de la niñez en sus complejos y cambiantes entornos. 


\section{Conclusiones}

El discurso de la calidad educativa ha provocado en las universidades ecuatorianas la búsqueda de mecanismos para mejorar sustancialmente los indicadores de gestión, siendo uno de ellos, la tasa de eficiencia terminal, la misma que se está incrementado significativamente en los últimos períodos académicos. Lamentablemente, este loable esfuerzo, en su afán de mostrar cifras y datos verificables, deja fuera algunos elementos de tipo cualitativo que para el caso de la formación investigativa son de extrema importancia. No hay duda que las universidades están obligadas a cumplir moral y legalmente con las normas referidas a la educación superior de los países en donde se encuentran ejecutando sus proyectos académicos, pero este cumplimiento debe ser analizado, razonado y ubicado en su respectivo contexto. El fin último de la universidad no está determinado por los indicadores planteados por las instancias de control; al contrario, éstos apenas son el punto de partida para otras metas mayores que la carrera y la universidad deben perseguir de acuerdo a su ideario institucional. Si se tiene presente esto, los indicadores serán asumidos en su justa dimensión y dejarán de convertirse en esa especie de pesado fardo que agobia a las universidades.

El problema de fondo que se esconde detrás de la experiencia de la Mención Parvularia parece ser la manera en que la carrera está afrontando el tema de la formación investigativa de sus estudiantes. Es evidente que el esquema anterior utilizado para el proceso de graduación de los estudiantes no respondía satisfactoriamente a las necesidades formativas y que ameritaba buscar soluciones efectivas que, a la vez que potenciaran la investigación, permitieran culminar en los tiempos previstos la formación profesional de los estudiantes. Pero también es evidente que los exámenes de grado tampoco resuelven el problema de la falta de investigación. Estas modalidades se corresponden con los esquemas de la universidad profesionalizante o universidad docente (Bellettini \& Ordóñez, 2013) que aún hoy proliferan en el país, pero ya no tienen cabida en el nuevo modelo de universidad de investigación. El cambio de matriz productiva y cognitiva que se plantea en el Ecuador se convierte en una buena oportunidad para que la Mención Parvularia reoriente la formación investigativa de sus estudiantes.

La capacidad investigativa para los docentes de educación inicial no es solamente uno de los tantos componentes del currículo, sino que en el escenario actual, se convierte en un elemento identitario de su profesión. En ese contexto, la formación investigativa debe ser fortalecida para que cumpla su cometido y una de las vías para que ello se concrete está en los denominados trabajos de titulación en donde se puedan aplicar los conocimientos, habilidades y valores propios de la actividad investigativa. Existen otros ejercicios y tareas académicas en el ciclo formativo de los estudiantes en que también pueden desarrollar estos elementos; pero, debido al modelo curricular que opera actualmente en la carrera, ninguno supera al trabajo de titulación, tanto más si éstos trabajos se vinculan a proyectos de investigación más amplios que son desarrollados por los centros de investigación y dirigidos por docentes experimentados.

\section{Referencias bibliográficas}

Abreu, José (2015). Síndrome todo menos tesis [All But Thesis Syndrome]. Daena: International Journal of Good Conscience, 10(2), 246-259. Recuperado 8 de abril de 2017, a partir de https://goo.gl/XVXRsC

Belletini, Orazio, \& Ordóñez, Andrea (2013). Ecuador: del país recursos al país conocimiento. Quito: Grupo FARO.

Buendía, Angélica (2007). El concepto de calidad: una construcción en la educación superior. Reencuentro, 50, 28-34. Recuperado 2 de diciembre de 2016, a partir de https://goo.gl/ohDtZI

Carr, Wilfred (2002). Una teoría para la educación. Hacia una investigación educativa crítica $\left(3 .^{a}\right.$ ed.). Madrid: Morata.

Carr, Wilfred, \& Kemmis, Stephen (1988). Teoría crítica de la enseñanza. La investigación-acción 
en la formación del profesorado. Barcelona: Martínez Roca.

Carreras, Llorenç, Eijo, Pilar, Estanytç, Assumpció, Gómez, María, Mir, Victoria, Ojeda, Felipa., .. Serrats, María (2009). Cómo educar en valores (15. ${ }^{\text {a }}$ ed.). Madrid: Narcea.

CEAASES (2015). Modelo de Evaluación Institucional de Universidades y Escuelas Politécnicas. Recuperado 10 de diciembre de 2016, a partir de https://goo.gl/T7mscQ

CES (2015a). Reglamento de régimen académico. Recuperado 18 de abril de 2017, a partir de https://goo.gl/M7Cs3h

CES (2015b). Propuesta curricular genérica de las carreras de educación. Recuperado 28 de febrero de 2015, a partir de https://goo.gl/aoSjNT

Chirino, María (2002). Perfeccionamiento de la formación inicial investigativa de los profesionales de la educación. La Habana: Instituto superior pedagógico Enrique José Varona.

COMEVAL (2014). Informe de evaluación de resultados de aprendizaje. Quito: UPS.

COMEVAL (2015). Informe evaluación de resultados del aprendizaje. Quito: UPS.

CONEA (2009). Evaluación de desempeño institucional de las universidades y escuelas politécnicas del Ecuador. Recuperado 4 de septiembre de 2013, a partir de https://goo.gl/weYSfM

Díaz-Barriga, Frida, \& Hernández, Gerardo (2010). Estrategias docentes para un aprendizaje significativo (3. a ed.). México: McGraw-Hill.

Esper, Maricarmen (2008). Cómo educar en valores éticos (2. ${ }^{\mathrm{a}}$ ed.). México: Trillas.

Fabara, Eduardo (2013). Estado del arte de la formación docente en el Ecuador. Quito. Recuperado $18 \mathrm{de}$ abril de 2017, a partir de https://goo.gl/WeXEi2

Ferrer, Yaritza, \& Malaver, Mara (2000). Factores que inciden en el síndrome TMT en las maestrías de la Universidad de Zulía [Factors affecting the All but Thesys Syndrome (TMT) in the Masters Degree Programs of the University Zulía]. Opción, 16(31), 112-129. Recuperado 18 de abril de 2017, a partir de https://goo.gl/9vChHG

Freire, Paulo (2001). Política y educación. México: Siglo XXI.

García, Gilberto (2010). La formación investigativa del educador. Aportes e impacto. La Habana: Enrique José Varona.
Gascón, Yamila (2008). El síndrome de todo menos tesis como factor influyente en la labor investigativa [The syndrome of everything but thesis "TMT" as influential factor in the research work]. Recuperado 26 de agosto de 2013, a partir de https://goo.gl/HhlKUo

Giroux, Henry (1990). Los profesores como intelectuales. Hacia una pedagogía critica del aprendizaje (1ra ed.). Barcelona: Paidós.

Guevara, Carlos, \& Viera, Aixa (2009). Disminución del sindrome TMT. Presentado en 7th Latin American and Caribbean Conference for Engineering and Technology. Recuperado 13 de noviembre 2013, a partir de https://goo.gl/tBofrL

Guzmán, Jesús (2003). Los claroscuros de la educación basada en competencias (EBC). Revista Nueva Antropología, 19(62), 143-162. Recuperado 16 de abril de 2017, a partir de https://goo.gl/tlahs6

Larrea, Elizabeth (2014). El currículo de la educación superior desde la complejidad sistémica. Recuperado 18 de abril de 2017, a partir de https://goo.gl/4hgwIu

Larrea, Elizabeth (2016). Unidad curricular de titulación. Recuperado 18 de abril de 2017, a partir de https://goo.gl/ikIei3

McLaren, P. (1994). Pedagogía crítica y cultura depredadora. Políticas de oposición en la era posmoderna. Barcelona: Paidós.

Medina, Antonio, \& Salvador, Francisco (2009). Didáctica general (2. ${ }^{\mathrm{a}}$ ed.). Madrid: Pearson Educación.

Ministerio de Educación de Ecuador (2014). Currículo educación inicial 2014. Quito.

Ordóñez, Jacinto (2002). Pedagogía crítica y educación superior. Educación, 26(2), 185-196. Recuperado 11 de abril de 2015, a partir de https://goo.gl/nsYf2f

Paulo, Alfredo, \& Chirino, María (2012). El desarrollo de habilidades investigativas en las universidades de ciencias pedagógicas de Cuba y Bié (Angola). Presentado en VIII Congreso Internacional de Educación Superior, La Habana.

Ponce, Juan (2016). Educación superior en Iberoamérica. Informe Ecuador 2016. Recuperado 16 de abril de 2017, a partir de https://goo.gl/jwfa5Q 
Proyecto Tuning (2007). Informe final del Proyecto Tuning América Latina: Reflexiones y perspectivas de la Educación Superior en América Latina. Bilbao: Universidad de Deusto.

Quintero-Corzo, Josefina, Molina, Ancízar, \& Munévar Quintero, Fabio (2008). Semilleros de investigación: una estrategia para la formación de investigadores [Research Seed Beds: A Research Training Strategy]. Educación y Educadores, 11(1), 31-42. Recuperado 14 de septiembre de 2013, a partir de https://goo.gl/bW2OKI

Ramírez, Roberto (2008). La pedagogía crítica. Una manera ética de generar procesos educativos. [Critical Pedagogy An ethical way to generate educational processes] Folios, (28), 108-119. Recuperado 22 de agosto de 2013, a partir de https://goo.gl/xrqLY

Rojas, Cristian, \& Aguirre, Sebastián (2015). La formación investigativa en la educación superior en América Latina y el Caribe: una aproximación a su estado del arte [Research Training in Higher Education in Latin America and the Caribbean: an approximation to its state of the art]. Revista Eleuthera, 12, 197-222.
Recuperado 23 de septiembre de 2016, a partir de https://goo.gl/76Ct3w

Schön, Donald (1992). La formación de profesionales reflexivos. Hacia un nuevo diseño de la enseñanza y el aprendizaje en las profesiones. Barcelona: Paidós.

SENPLADES (2013). Plan Nacional del Buen Vivir 2013-2017. Recuperado 18 de abril de 2017, a partir de https://goo.gl/SkbP5F)

UNESCO (1998). Conferencia Mundial sobre la Educación Superior 1998: La educación superior en el siglo XXI. Visión y acción. Recuperado 5 de enero de 2015, a partir de https://goo.gl/d1vZMC

UPS (2009). Carta de navegación de la Universidad Politécnica Salesiana. Recuperado 18 de abril de 2017, a partir de https://goo.gl/Fp5jsY

UPS (2013). La UPS en cifras 2013. Recuperado 18 de octubre de 2014, a partir de https://goo.gl/55rV2E

UPS (2015). La UPS en cifras 2015. Recuperado 18 de abril de 2017, a partir de https://goo.gl/ Kfumi0

UPS (2016). Proyecto curricular de la carrera de Educación Inicial. 